Original Article

\title{
Factors characterizing gait performance of patients before and soon after knee arthroplasty
}

\author{
Hideyuki Ito, $\mathrm{MS}^{1,2)^{*}}$, Kiyoshi Ichihara, MD, $\mathrm{PhD}^{2)}$, Kotaro Tamari, $\mathrm{PhD}^{3)}$, \\ Tetsuya Amano, $\mathrm{PhD}^{4)}$, Shigeharu Tanaka, $\mathrm{PhD}^{5)}$, Shigehiro Uchida, MS ${ }^{6}$ ) \\ 1) Department of Rehabilitation, Faculty of Wakayama Health Care Sciences, Takarazuka University of \\ Medical and Health Care: 2252 Nakanoshima, Wakayama, Wakayama 640-8392, Japan \\ 2) Department of Clinical Laboratory Sciences, Faculty of Health Sciences, Yamaguchi University \\ Graduate School of Medicine, Japan \\ 3) Department of Physical Therapy, Faculty of Health and Medical Science, Teikyo Heisei University, \\ Japan \\ 4) Department of Physical Therapy, Faculty of Health and Medical Sciences, Tokoha University, Japan \\ 5) School of Rehabilitation, Faculty of Health and Social Services, Kanagawa University of Human \\ Services, Japan \\ 6) Department of Rehabilitation, Faculty of Rehabilitation, Hiroshima International University, Japan
}

\begin{abstract}
Purpose] For monitoring patients with knee osteoarthritis undergoing knee arthroplasty, the Timed Up and Go and maximum walking speed tests are commonly used. To provide appropriate peri-surgical rehabilitation, we evaluated the factors associated with postsurgical changes in Timed Up and Go and maximum walking speed results. [Participants and Methods] We enrolled 545 knee osteoarthritis patients undergoing either of the following knee arthroplasties: conventional total knee arthroplasty, minimally invasive total knee arthroplasty, and unicompartmental knee arthroplasty. Comfortable Timed Up and Go, maximum Timed Up and Go, and maximum walking speed were measured 2 weeks before and soon after surgery. Factors (gender, age, and surgical mode) that might influence changes in test results were evaluated by multiple regression analysis and a two-factor stratification diagram. [Results] Multiple regression analysis revealed that postsurgical changes in comfortable/maximum Timed Up and Go and maximum walking speed results were associated with age and surgical mode after adjustment for preoperative values. Two-factor diagrams showed that the older the patient, the greater was the slowdown in the Timed Up and Go test performed postoperatively. The levels of slowdown in the postoperative Timed Up and Go and maximum walking speed tests were the smallest in those who underwent conventional total knee arthroplasty, followed by those who underwent minimally invasive and unicompartmental knee arthroplasty. Among patients whose preoperative Timed Up and Go and maximum walking speed were slow, slowdown in Timed Up and Go was pronounced with age, and slowdown in maximum walking speed was higher in conventional total knee arthroplasty. [Conclusion] The changes in Timed Up and Go and maximum walking speed results 2 weeks after knee arthroplasty depended on age and surgical modes. These findings are relevant for the implementation of appropriate peri-surgical rehabilitation.

Key words: Multiple regression analysis, Age-related change, Surgical mode
\end{abstract}

(This article was submitted Nov. 24, 2020, and was accepted Dec. 15, 2020)

*Corresponding author. Hideyuki Ito (E-mail: h-ito@tumh.ac.jp)

(C2021 The Society of Physical Therapy Science. Published by IPEC Inc.

(c) $(-)$ This is an open-access article distributed under the terms of the Creative Commons Attribution Non-Commercial No Derivacc. ${ }_{\mathrm{BY}} \mathrm{NC}_{\mathrm{ND}}$ tives (by-nc-nd) License. (CC-BY-NC-ND 4.0: https://creativecommons.org/licenses/by-nc-nd/4.0/) 


\section{INTRODUCTION}

Osteoarthritis (OA) of the knee, as reported in a large-scale resident cohort study, has an extremely high prevalence. The number of patients with knee OA is estimated to be 25.3 million, of whom approximately 8 million are assumed to have symptoms ${ }^{1)}$. If no improvements in pain or motor function can be seen in patients with knee OA, total knee arthroplasty (TKA) or unicompartmental knee arthroplasty (UKA) are performed, with the total number of such operations reaching 82,000 per year in $\mathrm{Japan}^{2}$.

As important parameters in evaluating the abilities of patients after knee arthroplasty, Dobson et al. ${ }^{3)}$ recommend measurements of Timed Up and Go (TUG) and the maximum walking speed (MWS) in grasping an overall ability of walking and moving. Bade et al. ${ }^{4}$ ) evaluated 119 post-TKA patients by measuring TUG before and six months after surgery. The results showed that individuals whose TUG was more than 10.1 seconds and who were older than 72 years prior to surgery showed a decline in motor function six months after surgery. By evaluating 64 patients who had undergone TKA (32 men and 32 women), Bade et al. ${ }^{5)}$ also performed the TUG test before surgery and during acute stages, as well as a 6-minute walking test six months after surgery. They reported that the TUG scores before surgery and during the acute stages were related to long-term postoperative 6-minute walking distance. Taniguchi et al. ${ }^{6}$ ) measured physical function, such as TUG and muscle strength, of 81 post-TKA patients ( 8 males and 73 females) as well as physical activity volume, before surgery and one and six months post-surgery. They reported that the TUG scores at one month after surgery predicted physical activity volume six months after surgery. In other words, walking and moving abilities, such as TUG, before and in short-term after surgery, are important indicators, since they predict long-term postoperative motor function and physical activity volume. Regarding time-serial changes in gait performance after TKA, Bade et al. ${ }^{7)}$ measured TUG in 24 TKA patients before, one, three, and six months after the surgery. They reported that TUG dropped significantly in one month after surgery, and it did not recover for a considerable period of time after the surgery.

Possible factors that may affect post-surgical abilities in walking and moving are gender, age, BMI, and surgical modalities. However, there has been no systematic evaluation of those factors in early post-surgical period despite its immense importance for proper management of peri-surgical rehabilitation.

Therefore, we conducted a multicenter joint study to investigate 545 knee OA patients undergoing knee arthroplasty for their motor functions including TUG and MWS before and two weeks after the surgery. Multifaceted analyses were performed to explore possible factors that may be associated with post-surgical changes in TUG and MWS values.

\section{PARTICIPANTS AND METHODS}

The participants used in this study were 545 knee OA patients (127 males and 418 females) in whom either TKA or UKA had been indicated for treatment at thirteen facilities that cooperated with this study (one facility in the Kinki area, five in the Chugoku area, four in the Shikoku area, and three in the Kyushu area of Japan). Of these 545 knee OA patients, 99 underwent conventional TKA (C-TKA), 342 underwent minimally invasive TKA surgery (MIS-TKA), and 104 underwent UKA. MIS-TKA involves a skin incision less than $5 \mathrm{~cm}^{8)}$, while UKA has less excision of the bone and is regarded as a technique featuring minimal invasion of the quadriceps ${ }^{9,10)}$.

Inclusion criteria were: (1) diagnosis of knee OA; and (2) indications for TKA and UKA. Exclusion criteria were: (1) neurological findings such as motor paralysis; (2) marked restrictions in range of motion as well as pain of joints other than the knees, and movement limitations in getting up and walking; and (3) cognitive and mental dysfunctions. The patients were managed according to prescribed clinical pathways, assuming the implementation of a similar rehabilitation program at all thirteen facilities, and were planned to be discharged 21 days after surgery. The rehabilitation program comprised: (1) a range of motion exercises of the knee joint; (2) muscle-strengthening exercises of the gluteus maximum, gluteus medius, and quadricep muscles; and (3) training in activities in daily living (getting up, walking, and going up and down the stairs).

The study was designed as a prospective cohort study aimed at analyzing the factors that affect the amount of change in gait performance in patients undergoing TKA. Measurements were taken before surgery and at two weeks postoperatively.

As medical attributes, the following parameters were investigated using a questionnaire and medical records: gender; age; BMI; severity of osteoarthritis of the knee and its K-L classification ${ }^{11,12)}$; presence/absence of regular exercise habits; disability of the nonoperative knee (unilateral and bilateral); and operative method (C-TKA, MIS-TKA, or UKA). Regular exercise was defined as having the habit of engaging in exercise more than twice a week and for more than half an hour each time.

In the TUG test, the starting position is sitting on a chair leaning against a backrest, with the hands resting on the thighs. Upon a start signal, a stopwatch measures the time for the participant to stand up from the chair, walk $3 \mathrm{~m}$, turn around, return to the chair, and sit down. Two methods were performed: comfortable TUG, which is performed at a comfortable walking speed; and maximum TUG, performed at maximum walking speed ${ }^{13)}$.

For maximum walking speed (MWS), the test was set up on a flat, non-slip, straight walkway, and $5 \mathrm{~m}$ was established as the measurement distance. Three meters before and after the measurement distance were reserved for acceleration and deceleration. Participants were instructed to walk safely and as quickly as possible, without running. Measurement began as soon as the participant either stepped on or passed the start line, and the walking time until he/she passed the end line was recorded using a stopwatch. MWS (m/s) was calculated using $5 \mathrm{~m} \div$ walking time $(\mathrm{s})^{14)}$. 
The change in TUG and MWS was calculated by subtracting the pre-operative value from the post-operative value. As a result, if a change in TUG $(\Delta T U G)$ was positive, TUG was prolonged after surgery, whereas a change in MWS $(\triangle M W S)$ was negative, MWS was decreased after surgery.

For statistical analysis, Wilcoxon signed rank test was used to compare the preoperative and postoperative values of TUG and MWS. To identify possible factors related to the amount of change $(\Delta)$ in TUG and MWS after the surgery, we performed the multiple regression analysis (MRA). The amount of change in the comfortable TUG, maximum TUG, and MWS between pre- and post-surgery were set as object variables, while the following parameters were prepared as explanatory variables: gender; age; BMI; presence/absence of regular exercise habits ( $\mathrm{Yes}=0, \mathrm{No}=1$ ); degree of severity (ordinal scale of $0-4$ in the $\mathrm{K}-\mathrm{L}$ classification) as well as disability of the nonoperative side of the knee (unilateral=0, bilateral $=1$ ); and operative methods (dummy variables representing MIS-TKA and UKA were created by setting C-TKA as a reference category).

In order to adjust for possible influence of preoperative values to the analysis, we deliberately introduced a preoperative variable corresponding to the object variable as a control variable. The most appropriate combination of explanatory variables was selected using a stepwise method. Because the sample size was large, we used the standard partial regression coefficient $(|\mathrm{rp}|)$, instead of the $\mathrm{p}$-value, as the criterion to determine the significance of each explanatory variable. $|\mathrm{rp}| \geq 0.1$ was regarded as the significant effect size ${ }^{15}$.

In addition, to confirm the relationship between an object variable and factors detected by MRA, a multi-way partitioning of object variable was performed and the results were graphically shown as a two-factor stratification diagram. For example, if $\triangle T U G$ was found associated with two factors: preoperative TUG and surgical mode, $\Delta T U G$ was first partitioned by the level of preoperative TUG, and then further partitioned by surgical mode to see the relative contribution of the two factors.

Ethical considerations, explanations, and consent were made in compliance with the Helsinki Declaration and Ethical Guidelines for Clinical Studies. We explained, in writing, the content of our research to the participants, ensured they fully understood, and obtained their written consent. The study was implemented on receipt of approval by Tokoha University's Ethics Committee (Approval No.: Revised-2018-501H).

\section{RESULTS}

The study included 127 men and 418 women, with mean \pm SD ages of $74.4 \pm 8.0$ years and $74.2 \pm 7.6$ years, respectively. The BMI of men was $25.3 \pm 3.6 \mathrm{~kg} / \mathrm{m}^{2}$, and that of women was $25.3 \pm 3.6 \mathrm{~kg} / \mathrm{m}^{2}$. A total of $176(32.3 \%)$ participants exercised regularly, and 369 (67.7\%) did not. According to the K-L classification of severity, 29 (5.3\%) participants were grade 2, 252 $(46.2 \%)$ were grade 3, and $264(48.4 \%)$ were grade 4 . In terms of impediments to the non-operated knee, $205(37.6 \%)$ were unilateral, and $340(62.4 \%)$ were bilateral. As the operative method, $99(18.1 \%)$ underwent C-TKA, $342(62.8 \%)$ underwent MIS-TKA, and $101(18.5 \%)$ underwent UKA.

The median of the comfortable TUG decreased significantly after the surgery from $12.9 \mathrm{~s}$ before surgery to $14.9 \mathrm{~s}$ after surgery $(\mathrm{p}<0.0001)$, and the median of maximum TUG decreased from $10.4 \mathrm{~s}$ before surgery to $12.1 \mathrm{~s}$ after surgery $(p<0.0001)$ (Fig. 1). The median of MWS also decreased significantly from $1.04 \mathrm{~m} / \mathrm{s}$ before surgery to $0.90 \mathrm{~m} / \mathrm{s}$ after surgery $(\mathrm{p}<0.0001)$ (Fig. 2).

A multiple regression analysis was performed to identify factors relating to $\triangle \mathrm{TUG}$ and $\triangle \mathrm{MWS}$. As shown in the upper block of Table 1, $\Delta$ comfortable TUG was significantly associated with age (rp=0.20), UKA $(-0.22)$, and MIS-TKA ( -0.15$)$. As shown in the middle block in Table $1, \Delta$ maximum TUG was significantly associated with age $(0.21)$, UKA $(-0.23)$, and MIS-TKA (-0.15). As shown in the bottom block of Table 1, $\Delta$ MWS was significantly associated with age (-0.19), UKA (0.24), and MIS-TKA (0.18).
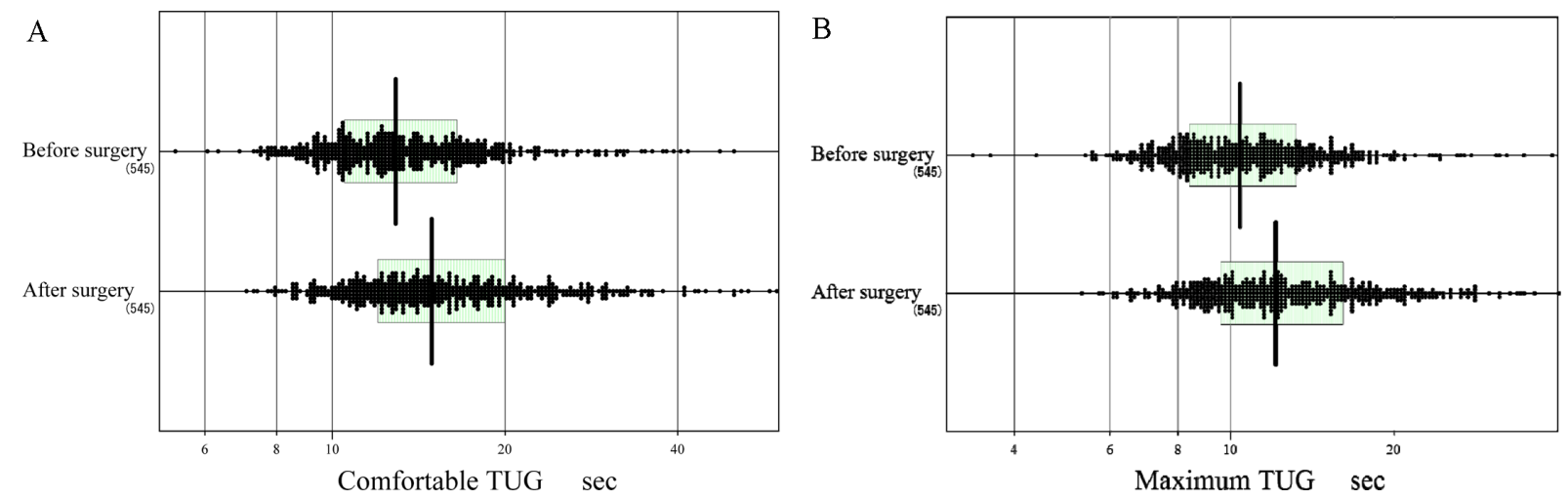

Fig. 1. Comparison diagram of Timed Up and Go (TUG) between pre- and post-surgery.

The measurement values of the comfortable TUG (Fig. 1A) and maximum TUG (Fig. 1B) are compared between pre- and postsurgery. The boxes and the line in the center show the central $50 \%$ range and the median for each group. 


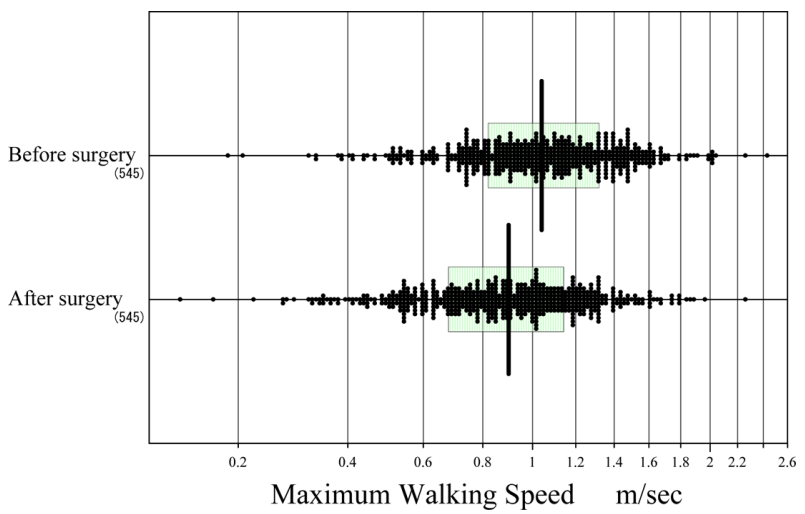

Fig. 2. Comparison diagram of maximum walking speed (MWS) between pre- and post-surgery. The measurement values of MWS are compared between pre- and post-surgery.

The boxes and the line in the center show the central $50 \%$ range and the median for each group.

Table 1. Multiple regression analysis exploring factors relating to post-surgical changes in TUG and MWS

\begin{tabular}{|c|c|c|c|c|c|}
\hline \multicolumn{6}{|c|}{ Object variable: $\Delta$ Comfortable TUG (After surgery-Before surgery) $n=545$} \\
\hline Explanatory variables & $\beta$ & $\operatorname{SE}(\beta)$ & $\operatorname{Std} \beta\left(r_{p}\right)$ & $\mathrm{df}$ & p-value \\
\hline Constant & 0.28 & 0.14 & & & \\
\hline Pre-operative Comfortable TUG & -0.31 & 0.03 & -0.43 & 540 & 0.000 \\
\hline Age & 0.01 & 0.00 & 0.20 & 540 & 0.000 \\
\hline UKA & -0.20 & 0.04 & -0.22 & 540 & 0.000 \\
\hline MIS-TKA & -0.11 & 0.04 & -0.15 & 540 & 0.002 \\
\hline \multicolumn{6}{|l|}{$<$ model fitness $>\mathrm{R}=0.4376, \mathrm{R}^{2}=0.1855$} \\
\hline \multicolumn{6}{|c|}{ Object variable: $\Delta$ Maximum TUG (After surgery-Before surgery) $n=545$} \\
\hline Explanatory variables & $\beta$ & $\operatorname{SE}(\beta)$ & $\operatorname{Std} \beta\left(r_{p}\right)$ & $\mathrm{df}$ & p-value \\
\hline Constant & 0.29 & 0.13 & & & \\
\hline Pre-operative Maximum TUG & -0.33 & 0.03 & -0.45 & 540 & 0.000 \\
\hline Age & 0.01 & 0.00 & 0.21 & 540 & 0.000 \\
\hline UKA & -0.19 & 0.04 & -0.23 & 540 & 0.000 \\
\hline MIS-TKA & -0.11 & 0.03 & -0.16 & 540 & 0.001 \\
\hline \multicolumn{6}{|l|}{$<$ model fitness $>\mathrm{R}=0.4532, \mathrm{R}^{2}=0.1995$} \\
\hline \multicolumn{6}{|c|}{ Object variable: $\Delta$ Maximum Walking Speed (After surgery-Before surgery) $n=545$} \\
\hline Explanatory variables & $\beta$ & $\mathrm{SE}(\beta)$ & $\operatorname{Std} \beta\left(r_{p}\right)$ & df & $\mathrm{p}$-value \\
\hline Constant & 0.87 & 0.13 & & & \\
\hline Pre-operative MWS & -0.49 & 0.03 & -0.58 & 539 & 0.000 \\
\hline Gender $($ female $=1)$ & -0.06 & 0.03 & -0.08 & 539 & 0.020 \\
\hline Age & -0.01 & 0.00 & -0.19 & 539 & 0.000 \\
\hline UKA & 0.18 & 0.04 & 0.24 & 539 & 0.000 \\
\hline MIS-TKA & 0.11 & 0.03 & 0.18 & 539 & 0.000 \\
\hline$<$ model fitness $>\mathrm{R}=0.5424, \mathrm{R}^{2}=0.2876$ & & & & & \\
\hline
\end{tabular}

These results are interpreted as follows: regarding $\triangle \mathrm{TUG}$, the higher the age, the steeper the postoperative decline of TUG, and the decline was smaller by UKA and MIS-TKA than by C-TKA. Likewise, regarding $\triangle \mathrm{MWS}$, the higher the age, the steeper the postoperative decrease in MWS, whereas the decrease was less by UKA and MIS-TKA than by C-TKA. It is of note that regardless of the object variable (preoperative value - postoperative value: $\Delta$ ), the preoperative values, which were set as control variable, were strongly related to the object variable.

For graphical interpretation of the MRA findings for each object variable, we drew two-factor stratification diagrams. In Fig. 3, $\Delta$ comfortable TUG test was compared according to preoperative TUG and age. The preoperative TUG was partitioned 

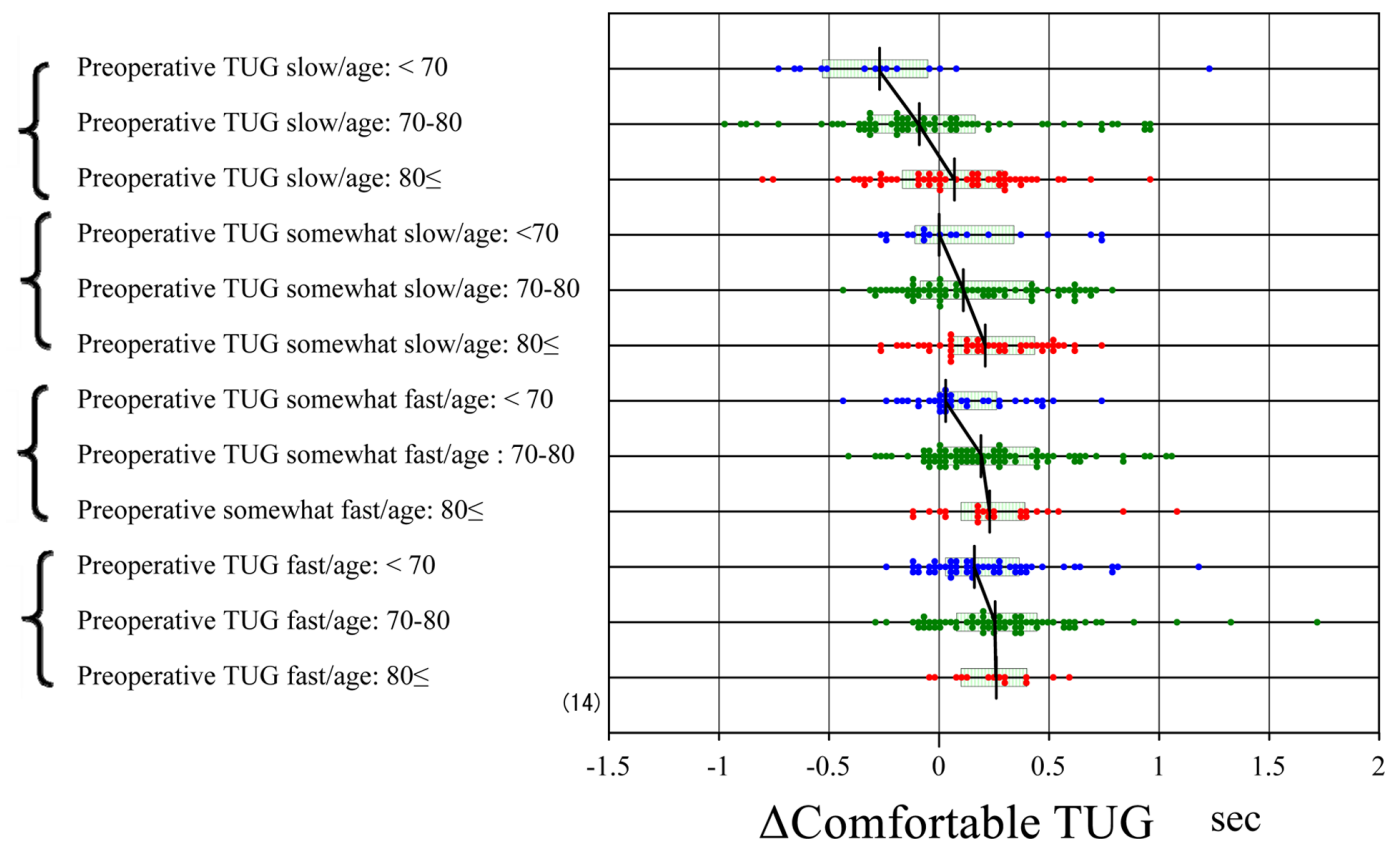

Fig. 3. A stratification diagram by preoperative values and by age vis-à-vis the amount of change in comfortable Timed Up and Go (TUG).

The amount of change in the comfortable TUG test is compared by stratifying preoperative values and age.

The boxes and the line in the center show the central $50 \%$ range and the median for each group.

The lines connecting the medians between age subgroups were drawn to visualize the magnitude of changes in values by age.

at 1st, 2nd, and 3rd quartiles to make four groups: "slow", "somewhat slow", "somewhat fast", and "fast", while the age was partitioned at 70 and 80 to make three groups: $<70,70-79, \leq 80$. The effect of age on $\Delta$ comfortable TUG was apparent for patients who showed "slow" preoperative TUG, but not in other groups. In Fig. 4, $\Delta$ comfortable TUG was partitioned by preoperative TUG and operative modes. $\Delta$ comfortable TUG was increased in the ascending order by UKA, MIS-TKA and C-TKA. This tendency was more pronounced in patients whose preoperative TUG was somewhat slow.

In Figs. 5 and 6, $\Delta$ maximum TUG was again partitioned by preoperative TUG group and by age-group or by operative modes. $\triangle$ maximum TUG was increased with age regardless of the preoperative TUG level, although it is more pronounced in patients with "slow" preoperative TUG. On the other hand, $\triangle$ maximum TUG was generally increased in the ascending order by UKA, MIS-TKA and C-TKA with its tendency more pronounced in patients with "somewhat slow" preoperative TUG.

In Figs. 7 and 8, $\Delta$ MWS was again partitioned by preoperative MWS group and by age-group or by operative modes. $\triangle$ MWS was slowed down by age regardless of preoperative MWS levels, although it was more pronounced in patients categorized as "somewhat slow" preoperative MWS. On the other hand, $\triangle$ MWS was generally slowed down in the ascending order by UKA, MIS-TKA and C-TKA with its tendency more pronounced in patients belonged to "slow", "somewhat slow", or "somewhat fast" preoperative MWS category.

\section{DISCUSSION}

Improvement in daily living after TKA and UKA depends on subsequent gait performance. However, it has been shown that gait performance soon after TKA surgery is poorer than before surgery, and that recovery takes time ${ }^{7)}$. No studies have been performed to evaluate factors that are related to postoperative changes in gait performance. On an occasion of conducting a multicenter follow-up study of 545 knee OA patients undergoing knee arthroplasty, we tried to explore possible factors that determine short-term postsurgical changes in TUG and MWS at two weeks. As a result, among clinical parameters examined, we found age, preoperative TUG or MWS, and operative modes were major factors determining the magnitude of post-arthroplasty changes in TUG and MWS. However, other clinical variables such as gender, BMI, severity of OA changes, habits of regular exercise were found irrelevant for the postoperative changes in gait performance.

As for our finding of age-related aggravation in post-surgical TUG (prolongation) and post-surgical MWS (slow-down), there has been no such a report to our knowledge. However, age-related changes in TUG and MWS have been well known. Shimada et al. ${ }^{16)}$ investigated the changes in TUG enrolling 959 elderly individuals (396 men and 563 women) and reported that TUG slowed with increased age in both men and women. Seino et al. $\left.{ }^{14}\right)$ investigated age- and gender-related differences 

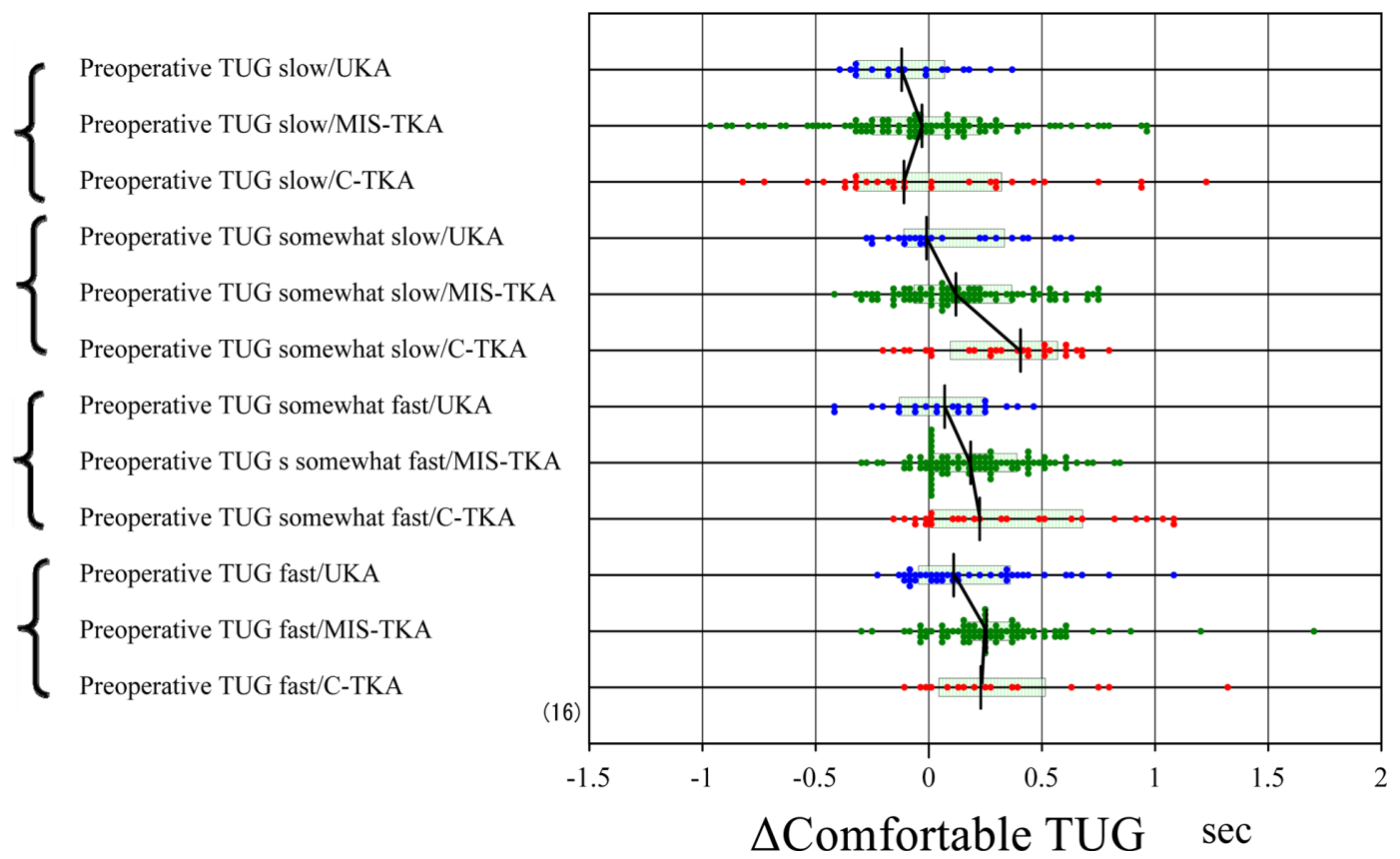

Fig. 4. Stratification diagram by preoperative values and by operative method vis-à-vis the amount of change in comfortable Timed Up and Go (TUG).

The amount of change in the comfortable TUG test is compared by stratifying preoperative values and operative methods.

The boxes and the line in the center show the central $50 \%$ range and the median for each group.

The lines connecting the medians between operative-mode subgroups were drawn to visualize the magnitude of changes in values by operative modes.

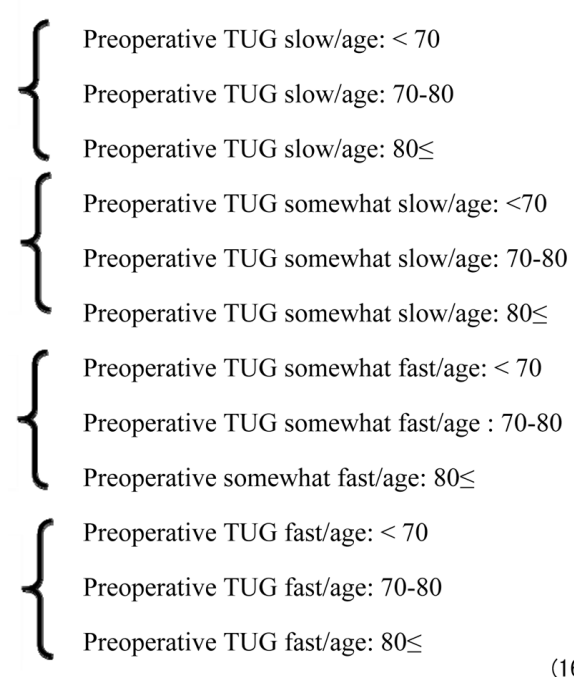

(16)

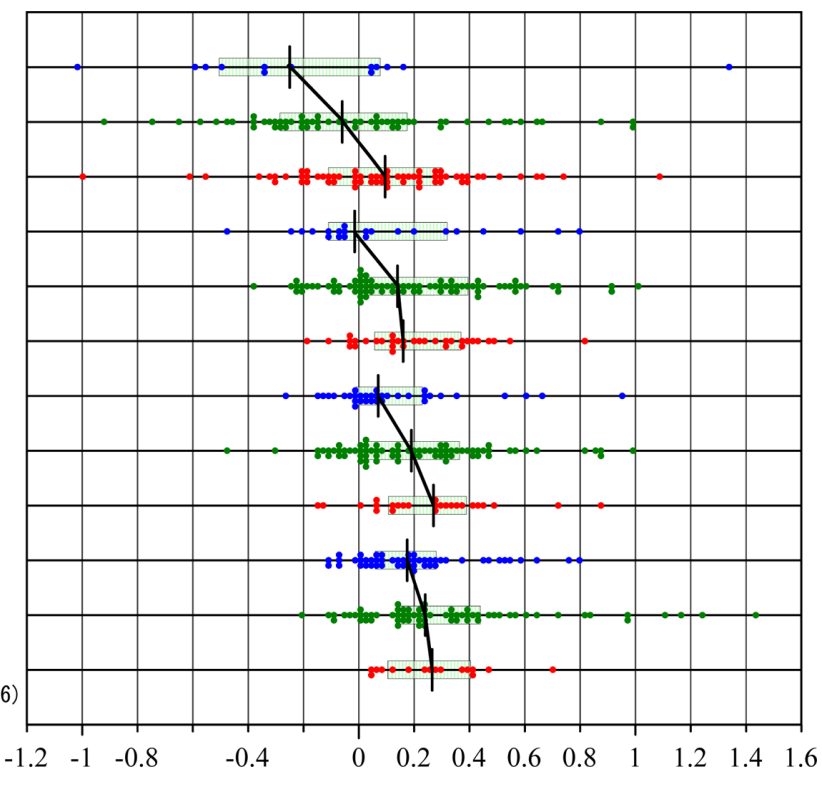

$\triangle$ Maximum TUG sec

Fig. 5. Stratification diagram by preoperative values and by age vis-à-vis the amount of change in maximum Timed Up and Go (TUG). The amount of change in the maximum TUG is compared by stratifying the preoperative values and age.

The boxes and the line in the center show the central 50\% range and the median for each group.

The lines connecting the medians between age subgroups were drawn to visualize the magnitude of changes in values by age. 

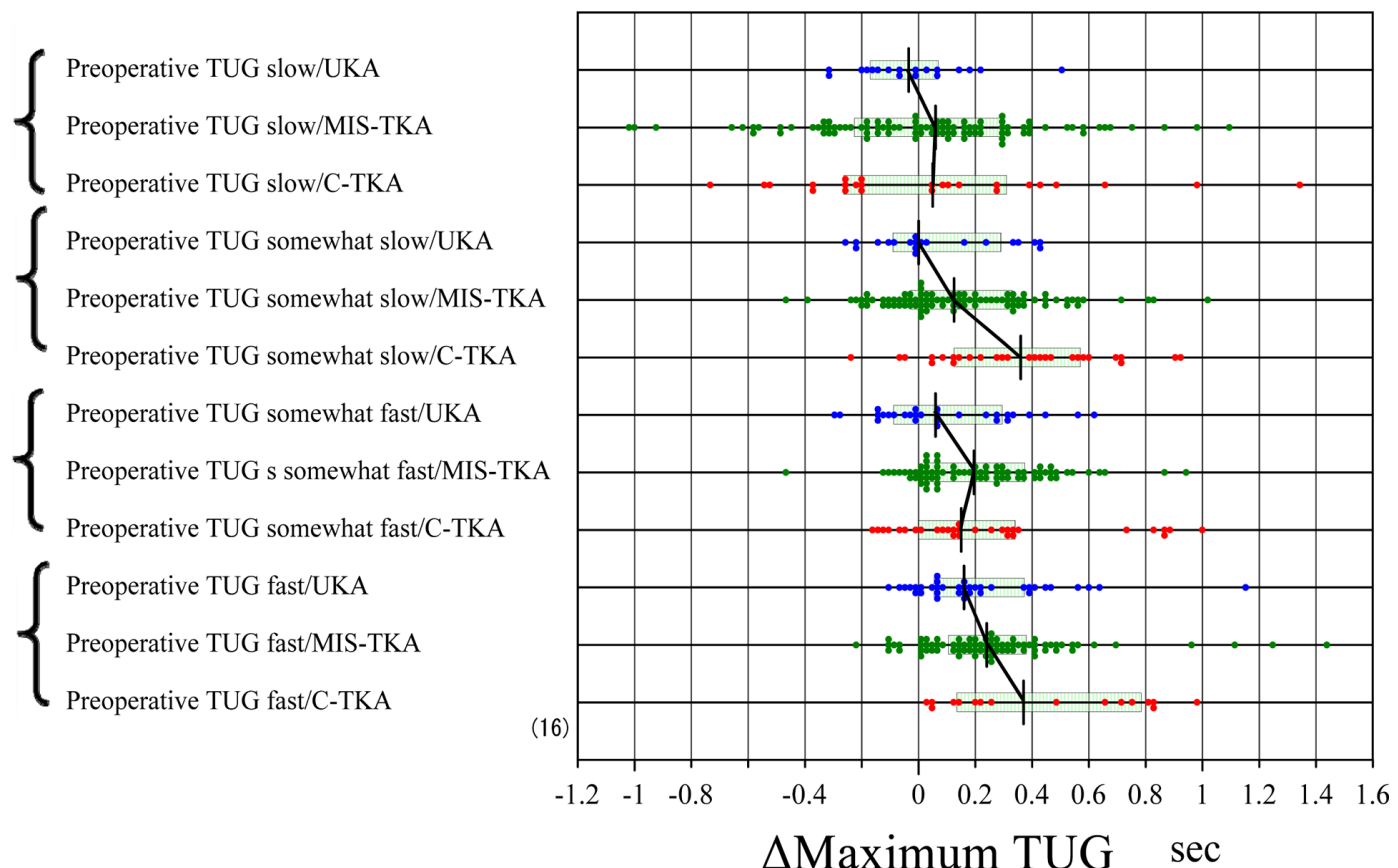

Fig. 6. Stratification diagram by preoperative values and by operative method vis-à-vis the amount of change in maximum Timed Up and Go (TUG).

The amount of change in maximum TUG is compared by stratifying preoperative values and operative methods.

The boxes and the line in the center show the central $50 \%$ range and the median for each group.

The lines connecting the medians between operative-mode subgroups were drawn to visualize the magnitude of changes in values by operative modes.
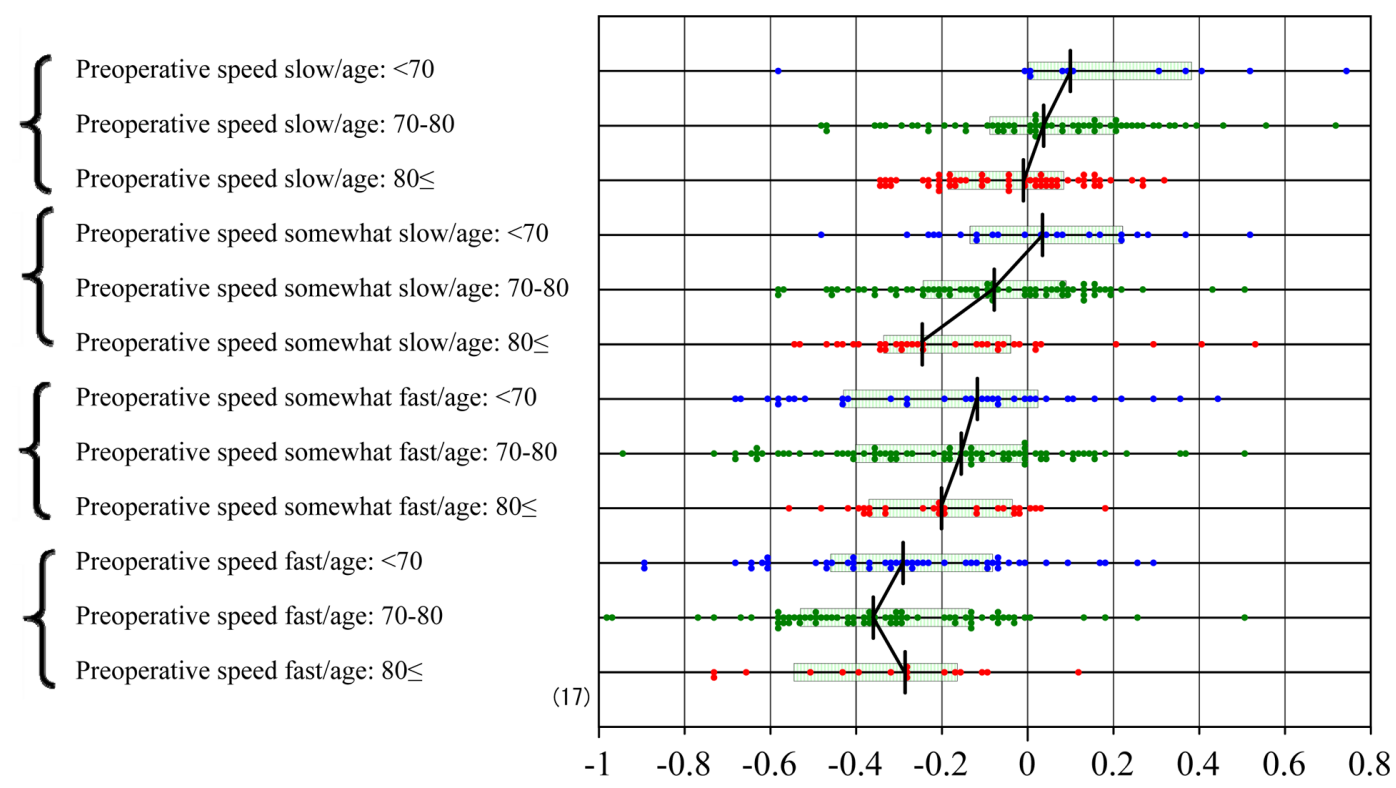

$\Delta$ Maximum Walking Speed $\mathrm{m} / \mathrm{sec}$

Fig. 7. Stratification diagram by preoperative values and by age vis-à-vis amount of change in of maximum walking speed (MWS). The amount of change in MWS is compared by stratifying the preoperative values and the and age.

The boxes and the line in the center show the central $50 \%$ range and the median for each group.

The lines connecting the medians between age subgroups were drawn to visualize the magnitude of changes in values by age. 

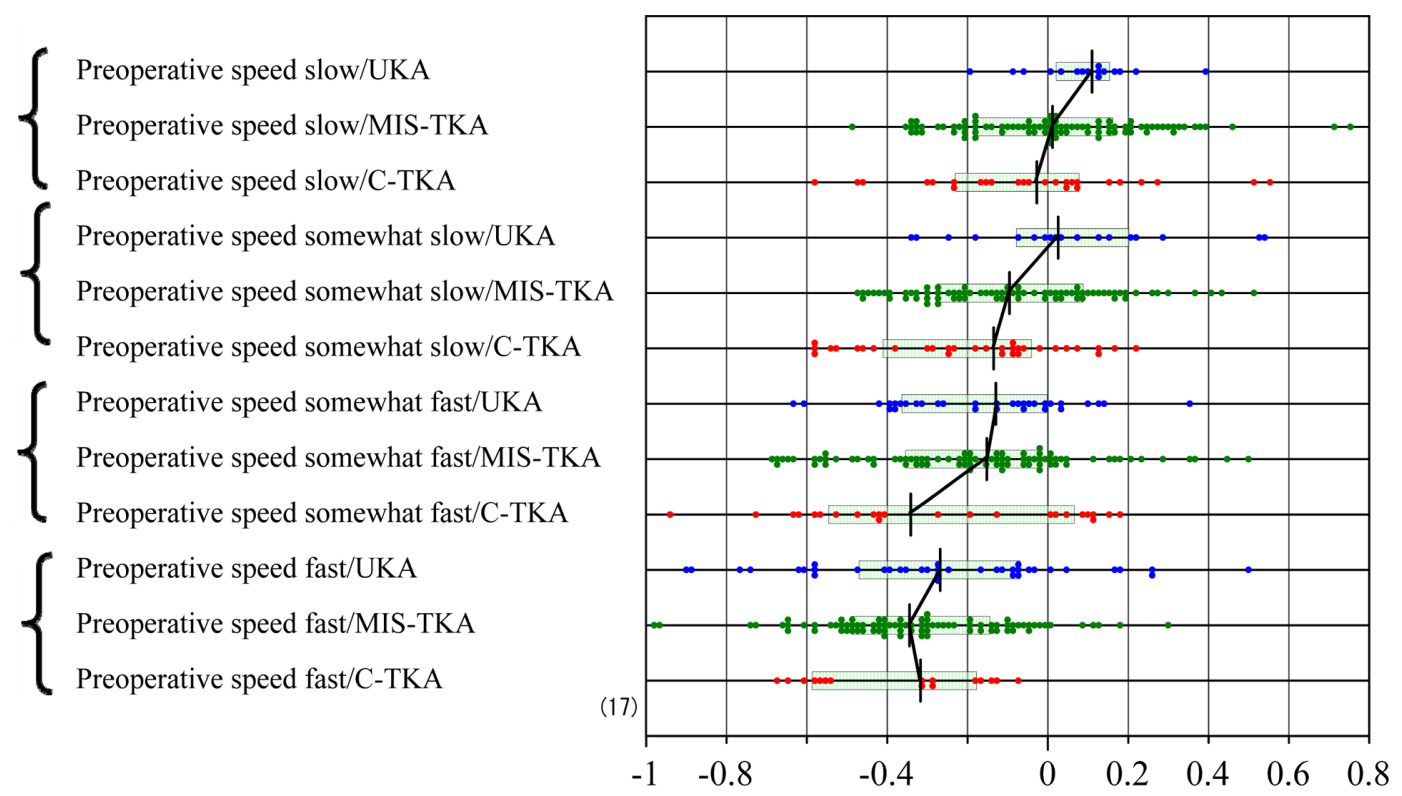

$\Delta$ Maximum Walking Speed $\mathrm{m} / \mathrm{sec}$

Fig. 8. Stratification diagram by preoperative values and by operative mode vis-à-vis amount of change in of maximum walking speed (MWS).

The amount of change in MWS is compared by stratifying the preoperative values and the operative method.

The boxes and the line in the center show the central $50 \%$ range and the median for each group.

The lines connecting the medians between operative-mode subgroups were drawn to visualize the magnitude of changes in values by operative modes.

in walking speed, using 4,683 healthy residents aged over 65 (2,168 men and 2,515 women) as their participants. Judged from these reports, our finding that the aggravation (slowing) of TUG and MWS immediately after the surgery is worse in patients with higher age, especially among those whose preoperative TUG were classified as "slow", is quite understandable.

Regarding our finding of lesser slow-down of walking ability (TUG and MWS) at two weeks after arthroplasty by the surgical modes of UKA and MIS-TKA compared to C-TKA, there have been many reports. Jones et al. ${ }^{17)}$ examined MWS and walking pattern of 12 TKA and 12 UKA patients at 12 months after the surgery, and compared the results with those of 121 healthy individuals. Their results showed that UKA patients walked faster than TKA patients, with articular and muscular movements that resembled those of healthy individuals, while TKA patients walked with decreased knee extension movements and avoidance of using quadriceps, resulting in a gait resembling that of patients suffering from anterior cruciate ligament injury. In other words, compared to C-TKA patients, UKA patients suffered from less injuries to the quadriceps and retained the knee-joint stability due to intact anterior cruciate ligament, and therefore appeared to allow walking like healthy individuals. Similar findings were also reported elsewhere ${ }^{18,19)}$. Therefore, this study not only confirmed that UKA results in less of a slowdown in TUG and MWS than C-TKA, but also demonstrated that this improved postsurgical walking ability by UKA occurred predominantly in patients whose preoperative MWS belonged to the "slow" or "somewhat slow" categories.

Regarding a beneficial post-surgical effect of MIS-TKA, Liebensteiner et al. ${ }^{20)}$ enrolled 17 MIS-TKA patients and 20 C-TKA patients and compared walking patterns at two months after surgery. They concluded that MIS-TKA patients did not show superior gait patterns. On the other hand, Tsuji et al. ${ }^{21)}$ measured physical activity with an accelerometer, targeting ten patients in MIS-TKA group and ten patients in C-TKA group, and made comparison at an acute stage of one and two weeks after the surgery. Their results showed that MIS-TKA patients retained a significantly higher level of physical activity. In summary, although the influence of MIS-TKA is minimal at two month postoperatively, during the acute stages, MIS-TKA provided an improved performance compared to C-TKA: i.e., a lesser slowdown in TUG and MWS. The finding is interpreted as due to minimal invasion to the quadriceps and less bleeding during surgery.

In this study, we demonstrated that beneficial effect of UKA is generally higher than MIS-TKA, and a more beneficial effect can be obtained among UKA or MIS-TKA patients whose presurgical MWS were in the categories of "slow" or "somewhat slow".

The limitation of this study was that we could evaluate the knee motor functions of patients undergoing knee arthroplasty only during a peri-surgical time of a short duration: before and two weeks after the arthroplasty. Therefore, post-surgical changes observed in the walking abilities are not applicable to predict a long-term changes in patients' capabilities, although 
our findings are relevant for proper management of peri-surgical rehabilitation customized to the clinical feature of each patient. Another limitation of the study was enrollment of insufficient number patients for more detailed evaluations of sources of post-surgical changes in walking abilities such as BMI, severity of OA, etc., with lack of statistical power for the analysis.

In summary, we conducted this prospective study of knee OA patients undergoing knee arthroplasty, aimed at exploring sources of variation of peri-surgical changes in walking abilities. Variable degrees of slow-down in comfortable/maximum TUG and MWS were observed at two weeks after the surgery, and the magnitude were found dependent on patients' age and surgical modes. With advancing age, the post-surgical slow-down of TUG and MWS was stronger especially among those whose preoperative walking abilities belonged to "slow" or "somewhat slow" category. The post-surgical slow-down was found prominent in patients underwent C-TKA, but was in a lesser degree among MIC-TKA patients, and in the least among UKA patients. The degree of slow-down was again dependent on pre-surgical walking abilities. These findings will be of practical importance to provide optimal peri-surgical rehabilitation in consideration of clinical features of each patient.

\section{Funding and Conflict of interest}

There is no conflict of interest in this study.

\section{REFERENCES}

1) Yoshimura N, Muraki S, Oka H, et al.: Prevalence of knee osteoarthritis, lumbar spondylosis, and osteoporosis in Japanese men and women: the research on osteoarthritis/osteoporosis against disability study. J Bone Miner Metab, 2009, 27: 620-628. [Medline] [CrossRef]

2) Keitaro Y, Tohru S: The current artificial knee joint market. Bone Jt Nerve, 2015, 15: 11-18.

3) Dobson F, Hinman RS, Roos EM, et al.: OARSI recommended performance-based tests to assess physical function in people diagnosed with hip or knee osteoarthritis. Osteoarthritis Cartilage, 2013, 21: 1042-1052. [Medline] [CrossRef]

4) Bade MJ, Wolfe P, Zeni JA, et al.: Predicting poor physical performance after total knee arthroplasty. J Orthop Res, 2012, 30: 1805-1810. [Medline] [CrossRef]

5) Bade MJ, Kittelson JM, Kohrt WM, et al.: Predicting functional performance and range of motion outcomes after total knee arthroplasty. Am J Phys Med Rehabil, 2014, 93: 579-585. [Medline] [CrossRef]

6) Taniguchi M, Sawano S, Kugo M, et al.: Physical activity promotes gait improvement in patients with total knee arthroplasty. J Arthroplasty, 2016, 31: 984-988. [Medline] [CrossRef]

7) Bade MJ, Kohrt WM, Stevens-Lapsley JE: Outcomes before and after total knee arthroplasty compared to healthy adults. J Orthop Sports Phys Ther, 2010, 40: 559-567. [Medline] [CrossRef]

8) Tria AJ Jr: Minimally invasive total knee arthroplasty: the importance of instrumentation. Orthop Clin North Am, 2004, 35: 227-234. [Medline] [CrossRef]

9) Murray DW, Goodfellow JW, O'Connor JJ: The Oxford medial unicompartmental arthroplasty: a ten-year survival study. J Bone Joint Surg Br, 1998, 80: 983-989. [Medline] [CrossRef]

10) Marmor L: Marmor modular knee in unicompartmental disease. Minimum four-year follow-up. J Bone Joint Surg Am, 1979, 61: 347-353. [Medline] [CrossRef]

11) Kessler S, Guenther KP, Puhl W: Scoring prevalence and severity in gonarthritis: the suitability of the Kellgren \& Lawrence scale. Clin Rheumatol, 1998, 17: 205-209. [Medline] [CrossRef]

12) Kellgren JH, Lawrence JS: Radiological assessment of osteo-arthrosis. Ann Rheum Dis, 1957, 16: 494-502. [Medline] [CrossRef]

13) Shumway-Cook A, Brauer S, Woollacott M: Predicting the probability for falls in community-dwelling older adults using the Timed Up \& Go Test. Phys Ther, 2000, 80: 896-903. [Medline] [CrossRef]

14) Seino S, Shinkai S, Fujiwara Y, et al. TMIG-LISA Research Group: Reference values and age and sex differences in physical performance measures for community-dwelling older Japanese: a pooled analysis of six cohort studies. PLoS One, 2014, 9: e99487. [Medline] [CrossRef]

15) Cohen J: A power primer. Psychol Bull, 1992, 112: 155-159. [Medline] [CrossRef]

16) Shimada H, Furuna T, Obuchi S, et al.: Timed Up \& Go test is a useful assessment tool for community health in elderly people. J Jpn Phys Ther Assoc, 2006, 33: $105-111$

17) Jones GG, Kotti M, Wiik AV, et al.: Gait comparison of unicompartmental and total knee arthroplasties with healthy controls. Bone Joint J, 2016, 98-B: 16-21. [Medline] [CrossRef]

18) Friesenbichler B, Item-Glatthorn JF, Wellauer V, et al.: Short-term functional advantages after medial unicompartmental versus total knee arthroplasty. Knee, 2018, 25: 638-643. [Medline] [CrossRef]

19) De Vroey H, Staes F, Vereecke E, et al.: Lower extremity gait kinematics outcomes after knee replacement demonstrate arthroplasty-specific differences between unicondylar and total knee arthroplasty: a pilot study. Gait Posture, 2019, 73: 299-304. [Medline] [CrossRef]

20) Liebensteiner MC, Thaler M, Giesinger JM, et al.: Mini-midvastus total knee arthroplasty does not result in superior gait pattern. Knee Surg Sports Traumatol Arthrosc, 2015, 23: 1699-1705. [Medline] [CrossRef]

21) Tsuji S, Tomita T, Fujii M, et al.: Is minimally invasive surgery-total knee arthroplasty truly less invasive than standard total knee arthroplasty? A quantitative evaluation. J Arthroplasty, 2010, 25: 970-976. [Medline] [CrossRef] 